\title{
Optimization of the surface plasmon resonance minimum detection algorithm for improvement of method sensitivity
}

\author{
R.V. Khrystosenko \\ V. Lashkaryov Institute of Semiconductor Physics, NAS of Ukraine, \\ 41, prospect Nauky, 03028 Kyiv, Ukraine; \\ E-mail:khristosenko@ukr.net
}

\begin{abstract}
Developed in this work is the information technology for improvement of the sensitivity inherent to the surface plasmon resonance (SPR) method by optimization of the resonance curve minimum detection algorithm. Simulation of SPR sensor response has been performed using the Fresnel equations with account of noise contribution and the Jones calculus. It has been demonstrated that using third-order polynomial approximation in the angular scanning range of $\theta_{\mathrm{SPR}} \pm 0.5^{\circ}$ with 100 experimental points improves the sensitivity of "Plasmon" series instruments by an order of magnitude.
\end{abstract}

Keywords: surface plasmon resonance, sensitivity, refractometry, measurement accuracy.

Manuscript received 29.04.15; revised version received 15.07.15; accepted for publication 03.09.15; published online 30.09.15.

\section{Introduction}

Surface plasmon resonance (SPR) phenomenon was discovered by R.W. Wood at the beginning of the $20^{\text {th }}$ century [1] and was widely used for studying the solid surfaces with single- and multilayer coatings [2,3]. The first publications on SPR application for gas and biomolecules detection appeared in 1982-1983 [4, 5]. Today SPR spectroscopy has evolved into a universal sensing method for studying surface molecular interactions in real time without labeling. With SPR, the researcher also obtains additional information on the investigated processes with high sensitivity and reliability, and the analysis consumes small sample quantities (of the order of microliters) [6].

Surface plasmons (SP) are the normal modes of charge density existing at the interface between metal and dielectric [7]. Resonant coupling of SP with photons linearly polarized in the plane of incidence by means of prism or diffraction grating leads to excitation of the strong electromagnetic wave that propagates along the metal-dielectric interface and exponentially decays in the direction of less optically dense medium at the distance of an order of the wavelength. Existence of the surface plasmon wave (SPW) is conditioned by dielectric properties of the metal, usually gold or silver, and the dielectric layer contacting with metal (the analyte that can be gas, liquid or solid substance), and manifests itself as a sharp minimum in the reflected light spectrum that is tracked by measurement of wavelength, angle of incidence or the reflected light intensity. Thus, the biochemical SPR sensor is a very sensitive thin-film refractometer that measures changes in the refraction index caused by adsorption or interaction of molecules on the metallic surface of the sensor.

The sensitivity of SPR sensors is defined as derivative of the measured parameter (resonant angle or wavelength) in respect to the parameter being determined (refraction index, adsorbed layer thickness, concentration etc.) [8]. Usually, sensitivity is expressed 
in the units of detected refraction index change (RIU). This quantity reflects the optical configuration implementation, measurement approach or data analysis algorithm, i.e., is determined by the particular instrument properties. E.g., the sensitivity of SPR sensors that utilize attenuated total reflection (ATR) prism for coupling is higher compared to grating coupling [9]. At present time, typical sensitivity of commercially available instruments is about $1 \cdot 10^{-5}-1 \cdot 10^{-6} \mathrm{RIU}$. In general, the sensitivity depends on many other parameters [10], including the technology used for sensitive element fabrication and sensor surface functionalization for specific applications. In this work, we present theoretical and experimental analysis of the method for improvement of the sensitivity in the compact computerized instrument of the "Plasmon" series by means of optimization of the SPR minimum detection algorithm.

\section{Measurement method}

For this research, a compact computerized instrument from "Plasmon" series developed at the V. Lashkaryov Institute of Semiconductor Physics of NAS of Ukraine was used [11]. The instrument principle of operation (Fig. 1) is based on prism (with refraction index $n=1.61$, base angle $65^{\circ}$, base side $(20 \times 20 \mathrm{~mm})$ in Kretschmann ATR configuration with SPW excitation in the thin gold film, which allows measuring the reflected $p$-polarized light intensity at the wavelength $\lambda=650 \mathrm{~nm}$ with changing the angle of light incidence [12]. The instrument enables to perform calibration for absolute angle values and provides registration of the full resonance curve with mechanical scanning the angle within 18 degrees of the limit in air (12 degrees in glass) and precision of 5 angular seconds.

SPW is excited in the thin gold film (1) deposited onto the glass substrate by thermal evaporation in

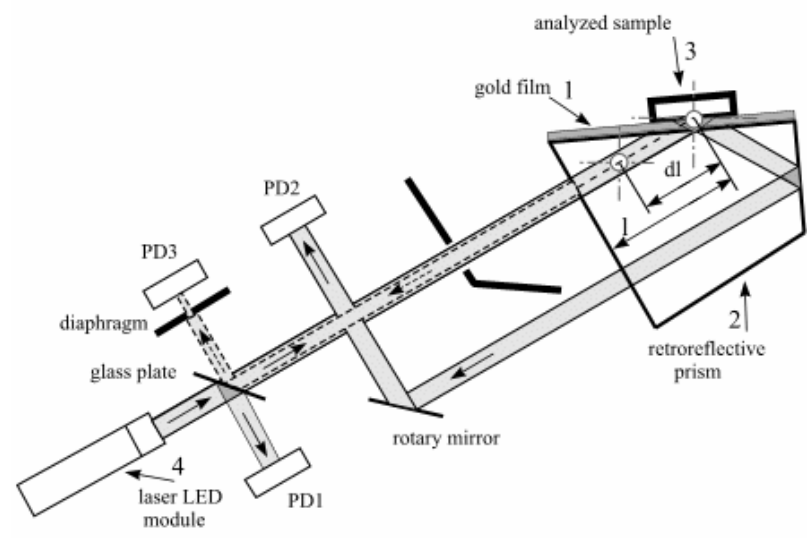

Fig. 1. Optical layout of the "Plasmon" instrument. vacuum and fixed on the face of glass retroreflective prism (2) due to immersion liquid with a respective refraction index. The upper side of the gold film contacts with the analyzed sample (3). Resonant coupling of excitation photons and electron plasma in the metal film surface took place due to the incidence of $p$-polarized light (4) from the prism bulk and scanning the inside surface of the metal film within the angular range exceeding the critical angle. Changing the angle of incidence of excitation beam is performed by prism rotation with the rotating platform driven by the step motor. To provide the contact of the working side of metal film to the liquid sample, a $100 \mu \mathrm{l}$ cell sealed with silicone rubber was used along with the peristaltic pump, which allowed to conduct measurements in the flowing sample mode with the controlled flow speed.

\subsection{Peculiarities of the "Plasmon" instrument optical configuration}

A laser LED module with optical system for parallel beam formation is used as the light source. It provides high precision of the incidence angle determination, limited only by the diffractive beam divergence, and allows to obtain correct values for the resonance curve in the wide range of angles.

Use of retroreflective prism with flat faces allows to minimize the incidence angle uncertainty and eliminate necessity for angular adjustment in registration during rotation by returning the beam after it reflects from the mirror at the back face of the prism. The beam crosses side face of the prism installed on the rotating platform and illuminates the part of the gold film placed in the sample cell. Three photodetectors are used for registration: PD1 monitors the intensity of incident light, PD2 registers the reflected light and PD3 is used for absolute angle calibration by detecting the moment of maximum light reflection from the front face of the prism through the diaphragm with a $100-\mu \mathrm{m}$ wide slit. Respective prism rotation angle is determined with enhanced precision by means of polynomial approximation, and based on that further absolute angular counts are calculated.

To obtain more accurate values of the reflected light intensity, the relation of geometric parameters of incident light beam and the sizes of instrument elements is optimized.

- Intensity of the reflected light is registered with photodetector, which size exceeds the section of the parallel beam, hence all the beam power is registered regardless of the light uniformity over the illuminated spot.

- To minimize the movement of the spot across the gold film surface, the axis of platform rotation does not coincide with the cell center, but is offset toward the light source by the distance $\delta l=(n-1) l$, where $l$ is the distance from the center of the cell to the point of incident beam entrance into the prism, $n$ is the prism refraction index. 
- The light source has the size of light emitting slit of $10 \times 0.2 \mu \mathrm{m}$ and produces the beam with divergence of $(10 \times 40)^{\circ}$, respectively, in the planes parallel and perpendicular to the emitting slit long axis. In this case, to provide registration of the entire power emitted by LED, the sample cell area has to be limited by the diameter of $8 \mathrm{~mm}$ and thickness of 50 to $100 \mu \mathrm{m}$, while its size in the instrument is $20 \times 20 \mathrm{~mm}$.

\subsection{Output signal of the "Plasmon" instrument}

The shape of the SPR curve and angular position of the minimum $\left(\theta_{S P R}\right)$ are determined by dielectric properties of metal and dielectric layer (analyte) contacting with the metal. As a result of adsorption of molecules at the gold surface or change in the refraction index of the gaseous/liquid analyte medium, the interface properties change correspondingly, and the resonance curve transformation occurs, which is registered by the "Plasmon" instrument as the output signal - shift of $\theta_{S P R}$ measured in angular seconds with time (kinetic SPR curve or sensogram). The resonance curve of reflection is registered by the control software at fixed time intervals, and the measurement results are being mathematically processed with the specially developed algorithm.

For the "Plasmon" series instruments, there are two main operation modes. The full SPR curve registration mode (when the angle measurement range is maximal) is usually used for subsequent fitting the experimental ant theoretic curves in order to determine the reflecting system parameters - thickness and optical constants of the investigated layers. Another operation mode, where scanning of the small part of SPR curve in direct vicinity of the minimum is performed, is used in applied tasks to track the changes in the SPR curve minimum position under the influence of factors such as molecules adsorption or change in the refraction index of analyzed medium. In this mode, the shift of the SPR curve minimum has to be tracked with high precision, which determines the instrument sensitivity.

The angular position of rotating platform in the "Plasmon" series instrument $\theta_{i}=\theta_{0}+\Delta \theta \cdot k(i)$ is determined by the step number $i$ of the rotation mechanism and initial angle $\theta_{0}$. Here, $\Delta \theta$ is the angular step, $k(i)$ - nonlinearity correction function that can be considered constant up to fractions of percent, and its value is determined by the instrument design. Each $i$ value corresponds to respective measured reflected beam intensity $I_{i}\left(\theta_{i}\right)=I_{0} R\left(\theta_{i}\right)$, where $R$ is reflectance.

\section{Measurement process simulation}

The theoretical reflectance curve for multilayer system "transparent substrate (glass) - metallic film (gold) dielectric (analyte) - surrounding medium" and $p$ polarized monochromatic incident light was calculated as a function of the angle of incidence by using Fresnel equations and Jones calculus [13], where the integral Fresnel reflectance is determined by the components of the optical constants matrix.

According to the Jones calculus formalism

$S=I_{10} L_{1} I_{12} L_{2} \ldots I_{(j-1) j} L_{j} \ldots L_{m} I_{m(m+1)}$,

where $I_{j m}$ is the Jones matrix for the interface between the layers $j$ and $m, L$ is the layer matrix:

$$
I_{j m}=\left[\begin{array}{cc}
1 & r_{j m} \\
r_{j m} & 1
\end{array}\right], L=\left[\begin{array}{cc}
e^{i \beta_{j}} & 0 \\
0 & e^{-i \beta_{j}}
\end{array}\right],
$$

$r_{j m}$ is reflectance for the corresponding interface, $\beta_{j}$ is the phase thickness of corresponding layer:

$\beta=2 N_{j} \pi \frac{d}{\lambda} \cos \theta_{j}$,

$N_{j}=n_{j}-i k_{j}$ is the complex refraction index of the layer, $\theta_{j}-$ angle of incidence within the $j$-th layer, $\lambda-$ wavelength, $d_{j}-$ layer thickness. Integral Fresnel reflectance of the multilayer structure for $p$-polarization is determined by the first column components of the effective optical constants matrix:

$$
R(\theta)=\left|R_{p}\right|=\left|S_{12 p}\right|=\frac{S_{21}}{S_{11}} .
$$

Since the classic Fresnel theory does not take into account physical effects leading to unpredictable transformations of resonance curve, experimental curve $R\left(\theta_{i}\right)$ was simulated by introducing noise signal of varying magnitude, i.e. by randomly changing the reflected light intensity in accordance with the following expression:

$$
R_{j}\left(\theta_{j}\right)=R_{j 0}\left(\theta_{j}\right)+\delta R(j) .
$$

Here, $R_{j 0}\left(\theta_{j}\right)$ is the theoretical SPR reflection curve, $R(j)$ - random function with a peak value of 1 , and $\delta-$ magnitude of the noise component constituting 0 to $10 \%$ of the maximum reflected light intensity value.

Computer simulation of the experimental SPR curve measurement process was performed by approximation of $R\left(\theta_{i}\right)$ function (where $i$ is the rotation mechanism step number) at $m$ points $x_{j}, y_{j} \quad(j=$ $1,2, \ldots, m)$ with polynomials of order $n$ using the least squares approach:

$$
y(x)=\sum_{i=0}^{n} a_{i} x^{i}
$$

Selection of the $x_{j}, y_{j}$ set for $R\left(\theta_{i}\right)$ function was made as based on the condition: $y_{1} \approx y_{m}, \quad x_{m}-x_{1}=\Delta \theta$, $x_{1}<\theta_{S P R}<x_{m}, m(\Delta \theta)$, where $\Delta \theta$ is the angular range of 
averaging (scanning), and the coefficients $a_{i}$ were determined from the condition:

$\min \sum_{j=0}^{m} w_{j}\left(y_{j}-\sum_{i=0}^{n} a_{i} x_{j}^{i}\right)^{2}$,

where $w_{j}$ is the weighting factor with the value $0 \leq w_{j} \leq 1$ for $j$ points.

Assuming that the angular position of simulated experimental SPR curve minimum is defined with a high accuracy, it was used as the reference for analysis of results of the experimental SPR curve approximation with polynomials of the order 2, 3, 4 and 5 with corresponding $a_{i}$. The angular scanning range $\Delta \theta$ was varied from $0.4^{\circ}$ through $2.7^{\circ}$.

To determine the polynomial minimum position, the following relations were used:

$$
\begin{array}{ll}
\boldsymbol{n}=\mathbf{2} & x_{\min (n=2)}=-a_{1} / 2 a_{2} ; \\
\boldsymbol{n}=\mathbf{3} & x_{\min (n=3)} \text { has two solutions }
\end{array}
$$
$\left(-a_{2} \pm \sqrt{a_{2}^{2}-3 a_{3} a_{1}}\right) / 3 a_{3}$, the value closest to $x_{\min (n=2)}$ was selected;

$\boldsymbol{n}=4,5 x_{\min (n=4,5)}$ is determined by minimization of (6) with the corresponding set of approximating polynomial coefficients $a_{i}$ within the neighborhood of $x_{\min (n=3)}$ using the algorithm described in [14].

As a result of performed calculations, it was shown that the SPR curve is optimally described by a parabola or a third order polynomial in the angular scanning range of $\left(\theta_{S P R} \pm 0.5^{\circ}\right)$, which is illustrated in Figs. 2 and 3. To decrease the time of analysis when investigating the SPR curve minimum position shift kinetics, the operation mode with scanning of a small portion of resonance curve (track mode) in the vicinity of minimum by the light incidence angle is specifically used.

The sensitivity of SPR instruments is determined by precision of determining the analyte refraction index $N_{P}$, while the output parameter is the change of angular position of reflection minimum $\theta_{S P R}$. Thus, the investigation of influence of approximating polynomial of the $2^{\text {nd }}$ and $3^{\text {rd }}$ order in the angular scanning range of $\pm 0.5^{\circ}$, instrument noise and the number of registered intensity values upon the accuracy of $N_{P}$ measurement was performed using the analysis of $\theta_{S P R}$ shift with the refraction index change by $1 \cdot 10^{-5}$ in two different areas: from 1.3300 to 1.3301 and from 1.3360 to 1.3361 . Computer simulation of the "Plasmon" series instrument response was performed for the instrument noise with the magnitude of $1 \%$ and $5 \%$ of maximum intensity value with the registered intensity values number of 64 and 400, and the magnitudes of absolute error and standard deviation of the SPR curve minimum position shift were estimated.

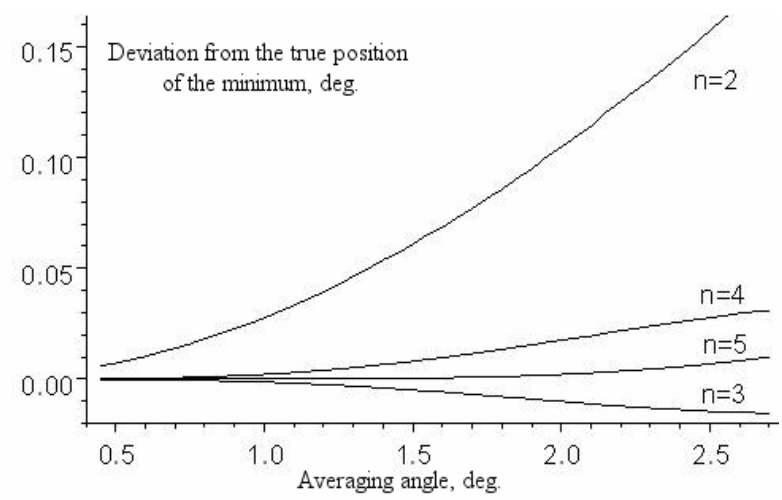

Fig. 2. Calculated values of $\theta_{S P R}$ for the $2 \mathrm{nd}, 3 \mathrm{rd}, 4 \mathrm{th}$ and $5^{\text {th }}$ order polynomials relative to the "true" position of SPR minimum depending on the angular range of averaging.

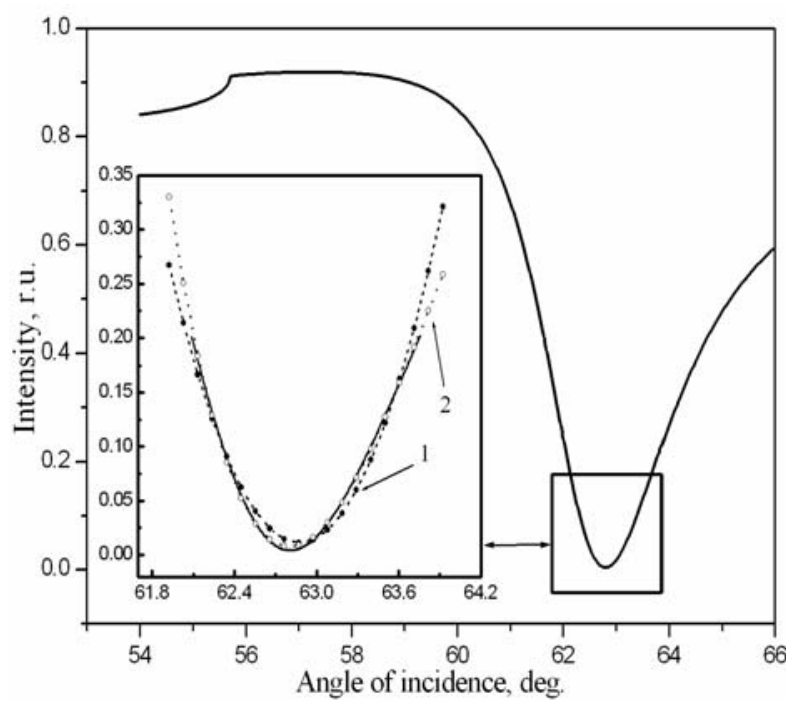

Fig. 3. Theoretical SPR curve (solid line) and quadratic (1) and cubic (2) polynomials (dotted lines) approximating it in the neighborhood of the minimum.

The simulation was performed as follows:

1. Function $R_{j 0}\left(\theta_{j}\right)$ was evaluated at 64 or 400 points from the interval of $\pm 0.5^{\circ}$ in the neighborhood of the minimum, corresponding to a certain value of $N_{p}$, then $\theta_{S P R}$ values were found from approximations with second- and third-order polynomials corresponding to each $N_{p}$ and the dependence $\theta_{S P R}\left(N_{p}\right)$ was built for each approximation.

2. Theoretical SPR curve was contaminated with noise with the magnitude of $1 \%$ and $5 \%$ of the maximum magnitude of $R$ in accordance with (5).

3. $\theta_{S P R 1}$ was found from the noisy curve.

4. Using the known dependence $\theta_{S P R}\left(N_{p}\right)$, the $N_{p 1}$ value was determined from $\theta_{S P R 1}$, as well as the noise-induced refraction index determination error $\delta N=N_{p}-N_{p 1}$.

Computation results are presented in Figs. 4 and 5 as well as in Table. From the analysis of obtained data the following conclusions can be made. 
Angular position of the minimum, deg.

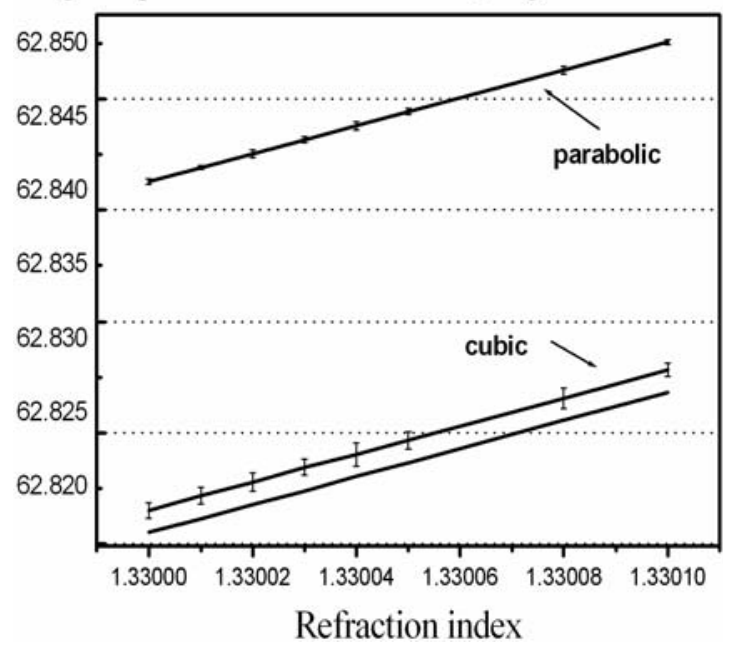

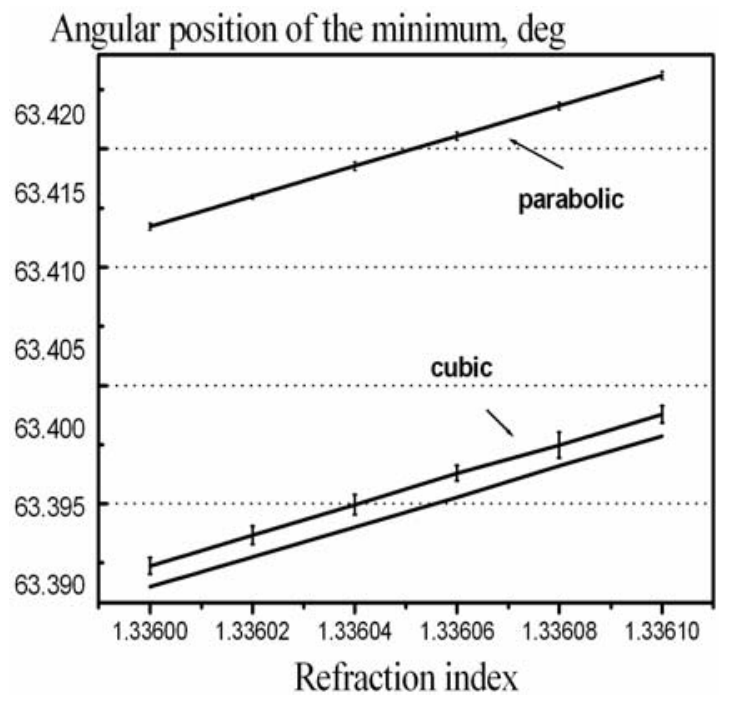

$b$

Fig. 4. Change of the angular position of the experimental SPR curve minimum (1), and the minimum of parabolic (2) and third order polynomial (3) approximations with the analyzed medium refraction index changing by $1 \cdot 10^{-5}$ within the ranges $1.33 \ldots 1.3301(a)$ and $1.336 \ldots 1.3361(b)$.

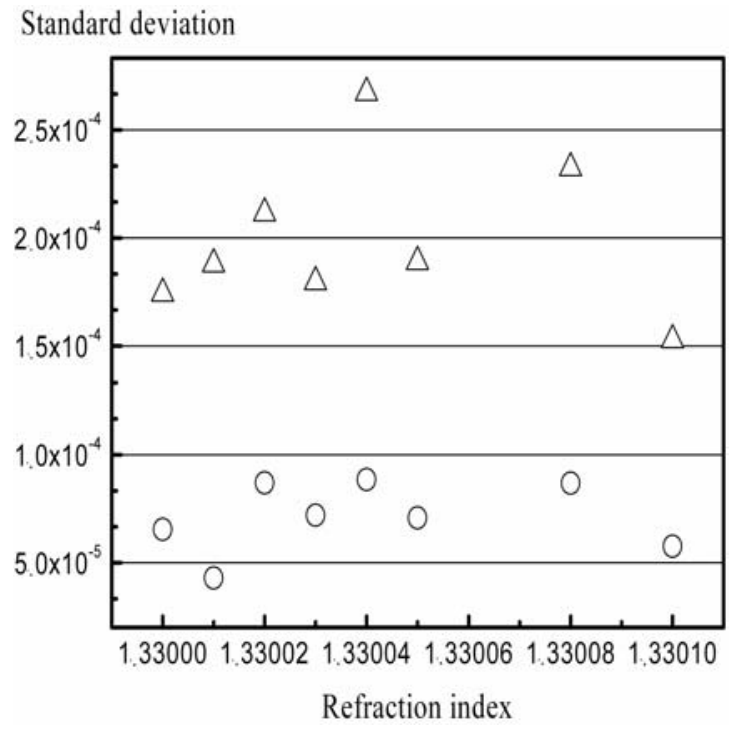

$a$

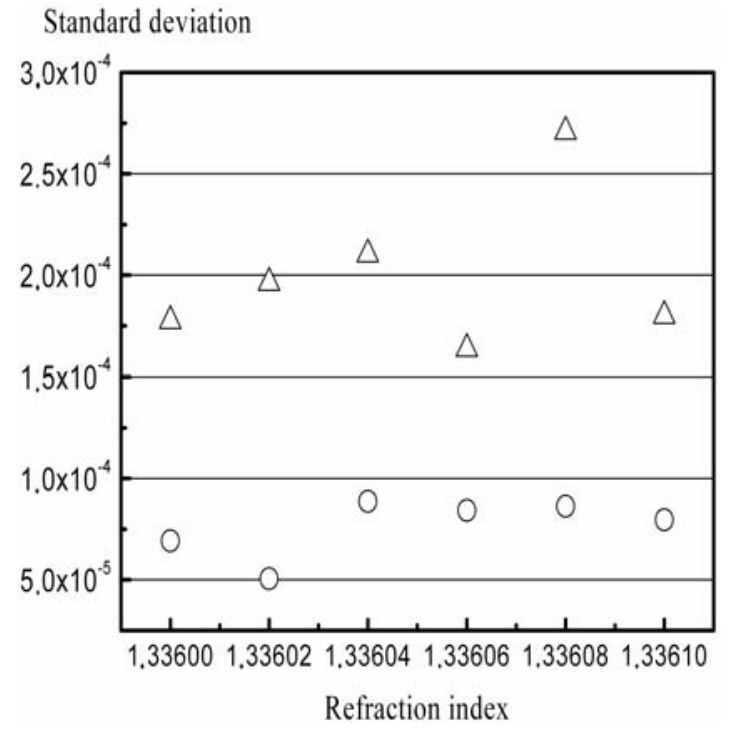

$b$

Fig. 5. Standard deviation of determination of the change in angular position of the minimum with parabolic (circles) and thirdorder polynomial (triangles) approximation of the experimental SPR curve and analyzed medium refraction index variation within the ranges $1.33 \ldots 1.3301(a)$ and $1.336 \ldots 1.3361(b)$.

Table. Influence of the noise and the number of measured points upon the error of determination of the analyzed medium refraction index.

\begin{tabular}{|l|c|c|c|c|c|c|}
\hline & \multicolumn{3}{|c|}{64 points, $\delta=1 \%$} & \multicolumn{3}{c|}{400 points, $\delta=1 \%$} \\
\cline { 2 - 7 } & $N_{p}$ & $N_{p 1}$ & $\delta N$ & $N_{p}$ & $N_{p 1}$ & $\delta N$ \\
\hline 1 & 1.330000 & 1.32999893 & $-1.07 \cdot 10^{-6}$ & 1.330000 & 1.32999948 & $-5.19 \cdot 10^{-7}$ \\
\hline 2 & 1.330010 & 1.33000880 & $-1.2 \cdot 10^{-6}$ & 1.330010 & 1.33000948 & $-5.2 \cdot 10^{-7}$ \\
\hline 3 & 1.330100 & 1.33009860 & $-1.4 \cdot 10^{-6}$ & 1.330100 & 1.33009931 & $-6.86 \cdot 10^{-7}$ \\
\hline 4 & 1.331000 & 1.33099847 & $-1.53 \cdot 10^{-6}$ & 1.331000 & 1.33099934 & $-6.65 \cdot 10^{-7}$ \\
\hline & \multicolumn{7}{|c|}{400 points, $\delta=5 \%$} \\
\hline 5 & 1.330000 & 1.32999618 & $-3.82 \cdot 10^{-6}$ & 1.330000 & 1.32999529 & $-4.71 \cdot 10^{-6}$ \\
\hline 6 & 1.330010 & 1.33000616 & $-3.84 \cdot 10^{-6}$ & 1.330010 & 1.33000531 & $-4.69 \cdot 10^{-6}$ \\
\hline 7 & 1.330100 & 1.33009628 & $-3.72 \cdot 10^{-6}$ & 1.330100 & 1.33009545 & $-4.55 \cdot 10^{-6}$ \\
\hline 8 & 1.331000 & 1.33099694 & $-3.06 \cdot 10^{-6}$ & 1.331000 & 1.33099543 & $-4.57 \cdot 10^{-6}$ \\
\hline
\end{tabular}

(C) 2015, V. Lashkaryov Institute of Semiconductor Physics, National Academy of Sciences of Ukraine 
Approximation with the third-order polynomial results in a smaller absolute error in obtained angular values and refraction index, however in presence of noise in the calculated curve parabolic approximation provides lower standard deviation. At the same time, noise with defined distribution practically does not influence determination of the refraction index, i.e. noise-induced error in the refraction index is small relative to systematic error caused by approximation of the calculated curve in the given angular range. Thus, parabolic approximation is appropriate to use in the studies of molecular reaction kinetics, and the thirdorder polynomial is more appropriate for determination of the refraction index absolute value. However, systematic error caused by approximation can be corrected, in which case the use of parabolic or thirdorder polynomial approximation for determination of $N$ is equally acceptable.

Presence of the noise in experimental curve with the magnitude of $1 \%$ leads to the errors in determination of the analyzed medium refraction index on the order of $10^{-6}$ with 64 measured points and approximately two times lower with 400 measured points, i.e. the increase of the registered intensity values number five-fold decreases the error in $N_{p}$ determination by factor of two.

The increase of the noise magnitude five-fold degrades the accuracy of $N_{p}$ determination approximately by factor of 3 (third column in Table) and this degradation virtually cannot be compensated by increasing the number of measured points. By contrast, increasing the number of measured points leads to the increased error (re. columns 3 and 6, rows 5-8). It means that, in the presence of large noise signal (about 5\%), increasing the measurement steps number is pointless.

\section{Experimental}

Experimental evaluation of the simulation results was performed with the "Plasmon" series instrument by measuring the difference in refraction indices of dry air (1.0003) and helium (1.0000296) that are permanent gases and do not adsorb on the gold film surface. The kinetic curve of the SPR minimum position change during sequential alternating injections of dry air and helium was registered employing the optimal algorithm of minimum detection: third-order approximating polynomial is used with 100 experimental points and angular scanning range of $\pm 0.5^{\circ}$ relative to the SPR resonant angle (track mode measurement).

Fig. 6 demonstrates the following measurement results: the resonance angle is $43.80213^{\circ} \pm 0.00156^{\circ}$ for air and $43.78883^{\circ} \pm 0.00169^{\circ}$ for helium. Difference in the SPR response is 47.87872 angular seconds, which corresponds to the difference in refraction indices of $0.000365 \pm 3.85726 \cdot 10^{-5}$. The obtained result agrees with the data known from the literature [15], which indicates correctness of the used approach in development of the new generation of SPR based instruments.

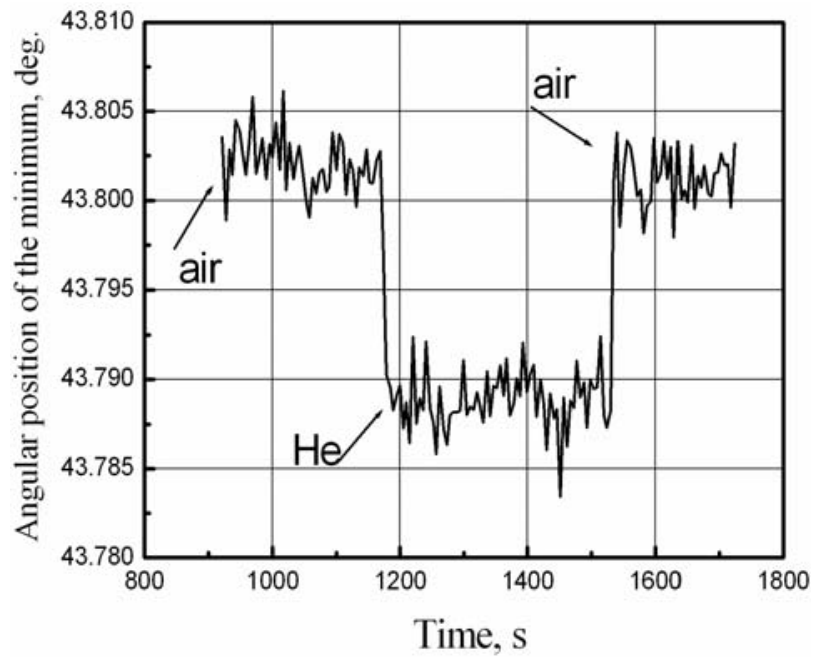

Fig. 6. Sensogram of the measurement of refraction indices difference with sequential injection of dry air and helium into the sample cell.

\section{Conclusions}

The work has been aimed at optimization of mathematical procedure for detection of the SPR curve minimum in the "Plasmon" series instrument, operation of which is based on SPW excitation in the thin gold film on retroreflective prism in Kretschmann configuration, providing the possibility for measurements of the full resonance curve with mechanical angular scanning within the 18 degrees limit on air (12 degrees in glass) and precision of 5 angular seconds.

Mathematical simulation of the thin-film optical sensor response for investigation of influence of the polynomial approximation order, angular scanning range and number of measured points has been performed with the use of Fresnel equations taking into account the noise contribution and Jones matrix calculus formalism. The absolute error magnitude and standard deviation have been estimated by evaluating the SPR curve minimum position shift with the analyte refraction index changing by $1 \cdot 10^{-5}$ in two different areas (from 1.3300 to 1.3301 and from 1.3360 to 1.3361 ) and simulated instrument noise with the magnitudes $1 \%$ and $5 \%$ of the maximum intensity, for 64 and 100 measured points.

It is shown that parabolic approximation is more appropriate for studying the molecular reaction kinetics, and third-order polynomial approximation - for determination of the absolute refraction index value. The optimal sensitivity of the "Plasmon" series instrument has been demonstrated using measurements of the difference in the refraction indices of dry air (1.0003) and helium (1.0000296) using the third-order approximating polynomial within the angular range $\left(\theta_{S P R} \pm 0.5^{\circ}\right)$ and 100 measured points. 


\section{References}

1. R.W. Wood // Phil. Mag. 23, p. 310-315 (1902).

2. I. Pockrand, J.D. Swalen, J.G. Gordon, M.R. Philpott, Surface plasmon spectroscopy of organic monolayer assemblies // Surf. Sci. 74, p. 237-244 (1978).

3. B. Rothenhausler, C. Duschi, W. Knoll, Plasmon surface polariton fields for the characterization of thin films // Thin Solid Films, 159, p. 323-330 (1988).

4. C. Nylander, B. Liedberg, T. Lind, Gas detection by means of surface plasmon resonance // Sensors and Actuators, 3, p. 79-88 (1982).

5. B. Liedberg, C. Nylander, I. Lundstrom, Surface plasmon resonance for gas detection and biosensing // Sensors and Actuators, 4, p. 299-304 (1983).

6. Surface Plasmon Resonance Based Sensors (Springer Series on Chemical Sensors and Biosensors) / Ed. by J. Homola. Springer-Verlag, Berlin-Heidelberg-New York, 2006.

7. Handbook of Surface Plasmon Resonance / Ed. by R.B.M. Schasfoort and Anna J. Tudos. Royal Society of Chemistry, Cambridge (UK), 2008.

8. R.P.H. Kooyman, H. Kolkman, J. van Gent, J. Greve, Surface plasmon resonance immunosensors: sensitivity considerations // Anal. Chim. Acta, 213, p. 35-45 (1988).
9. J. Homola, I. Koudela, S.S. Yee, Surface plasmon resonance sensors based on diffraction grating and prism couplers: sensitivity comparison // Sensors and Actuators B, 54, p. 16-24 (1999).

10. E.M. Yeatman, Resolution and sensitivity in surface plasmon microscopy and sensing // Biosensors Bioelectron. 11, p. 635-649 (1996).

11. Patent of Ukraine № 46018, CI7 G01N 21/55. Method of detecting and determining the concentration of biomolecules and molecular complexes and device for its realization / Yu.M. Shirshov, E.F. Venger, A.V. Prokhorovich, Yu.V. Ushenin, E.P. Matsas, V.I. Chegel, A.V. Samoilov - Invention application 22.10.1997, № 97105153; Publ. 15.05.2002, Bull. №5.

12. E. Kretschmann, H. Raether, Radiative decay of non-radiative surface plasmons excited by light // Z. Naturforschung, 23A, p. 2135-2136 (1968).

13. R.M.A. Azzam, N.M. Bashara, Ellipsometry and Polarized Light. North-Holland Publishing company, Amsterdam-New York-Oxford, 1977.

14. G.A. Korn, T.M. Korn, Mathematical Handbook for Scientists and Engineers. Daver Publications, Inc., Mineola, New York, 2000.

15. T. Grosges, B. Piraux, R. Shakeshaft, Rayleigh and Raman scattering from helium for wavelengths down to $16 \mathrm{~nm}$. Los Angeles: 1999 (Preprint/Physics Department,University of Southern California). 